\title{
A Gate-and-Switch Model for Head Orientation Behaviors in Caenorhabditis elegans
}

\author{
@i Marie-Hélène Ouellette, ${ }^{1}$ Melanie J. Desrochers, ${ }^{1}$ Ioana Gheta, ${ }^{1}{ }^{\circledR}$ Ryan Ramos, ${ }^{1}$ and ${ }^{\circledR}$ Michael \\ Hendricks ${ }^{1}$
}

https://doi.org/10.1523/ENEURO.0121-18.2018

${ }^{1}$ Department of Biology, McGill University, Montreal, Quebec H3A 1B1, Canada

\begin{abstract}
The nervous system seamlessly integrates perception and action. This ability is essential for stable representation of and appropriate responses to the external environment. How the sensorimotor integration underlying this ability occurs at the level of individual neurons is of keen interest. In Caenorhabditis elegans, RIA interneurons receive input from sensory pathways and have reciprocal connections with head motor neurons. RIA simultaneously encodes both head orientation and sensory stimuli, which may allow it to integrate these two signals to detect the spatial distribution of stimuli across head sweeps and generate directional head responses. Here, we show that blocking synaptic release from RIA disrupts head orientation behaviors in response to unilaterally presented stimuli. We found that sensory encoding in RIA is gated according to head orientation. This dependence on head orientation is independent of motor encoding in RIA, suggesting a second, posture-dependent pathway upstream of RIA. This gating mechanism may allow RIA to selectively attend to stimuli that are asymmetric across head sweeps. Attractive odor removal during head bends triggers rapid head withdrawal in the opposite direction. Unlike sensory encoding, this directional response is dependent on motor inputs to and synaptic output from RIA. Together, these results suggest that RIA is part of a sensorimotor pathway that is dynamically regulated according to head orientation at two levels: the first is a gate that filters sensory representations in RIA, and the second is a switch that routes RIA synaptic output to dorsal or ventral head motor neurons.
\end{abstract}

Key words: behavior; C. elegans; calcium signaling; sensorimotor integration

\section{Significance Statement}

Simple head orientation behaviors in Caenorhabditis elegans provide an opportunity to dissect sensorimotor integration at the cellular and subcellular levels. We describe a single interneuron that coordinates posture, sensation, and movement to detect the spatial distribution of stimuli and direct motor output to guide navigation behavior. Our findings underscore the fundamental role of neural mechanisms for integrating sensory input into ongoing behavior.

\section{Introduction}

Perception and action are intimately linked in the central nervous system. Our movements often have immediate sensory consequences, including changes in visual, proprioceptive, and mechanosensory inputs. Likewise,

Received March 28, 2018; accepted October 4, 2018; First published November 20, 2018.

The authors declare no competing financial interests.

Author contributions: M.H., M-H.O., and M.J.D. designed research; M.H., M-H.O., M.J.D., I.G., and R.R. performed research; M.H., M-H.O., M.J.D., I.G., and R.R. analyzed data; M.H. and M-H.O. wrote the paper. vocalization produces auditory self-stimuli, and inhalation can result in olfaction. To ensure accurate internal representations of the world, reafferent sensory stimulation caused by our own behavior must be processed and interpreted differently from other stimulus sources (Straka

This work was supported by McGill University (M.H. and M-H.O.), the National Science and Engineering Research Council Grant RGPIN/05117-2014 (to M.H.), the Canadian Foundation for Innovation Grant 32581 (to M.H.), and the Canada Research Chairs Program Grant 950-231541 (to M.H.). Some strains were provided by the Caenorhabditis Genetics Center, which is funded by National Institutes of Health Office of Research Infrastructure Programs 
et al., 2018). To make this distinction, our sensory systems must continuously predict the consequences of our own actions. This is in part accomplished via inputs from motor areas, called efference copies or corollary discharge (Crapse and Sommer, 2008). Through processes that are only partially understood, our senses are precisely filtered and modulated in real time to compensate for ongoing behaviors and the sensory consequences of our actions. While some of the neural circuits that underlie these functions have been anatomically characterized to varying degrees, the sites and molecular mechanisms of this type of sensorimotor integration remain elusive in all but a few cases. The nematode Caenorhabditis elegans, which has a completely mapped and largely invariant nervous system, quantifiable behaviors, and a suite of genetic and imaging tools, provides an opportunity to probe these events at cellular resolution and directly link them to behavior (White and Southgate, 1986; Calhoun and Murthy, 2017).

The primary mode of $C$. elegans navigation is a biased random walk consisting of bouts of forward movement punctuated by quasi-random reorientations achieved through clustered sequences of reversals and turns. Directional movement is achieved by measuring changes in sensory input over time during forward bouts and modulating reorientation probability accordingly (PierceShimomura et al., 1999). This behavior is directly analogous to the runs and tumbles of bacterial chemotaxis (Berg, 1975). C. elegans lie on their side, undulating in the dorsoventral plane as they crawl in a sinusoidal motion. Sensory structures located at the tip of the nose sweep back and forth as the head bends during forward movement. In contrast to the biased random walk, several navigation behaviors suggest that reafferent sensory input caused by head bending during locomotion is exploited as a way to sample the spatial distribution of environmental stimuli and guide navigation, a simple form of active sensing. For example, in radial temperature gradients, C. elegans will crawl in precisely curved trajectories to stay within a favored temperature range, a behavior called isothermal tracking (Hedgecock and Russell, 1975). Likewise, when crawling in a chemoattractant gradient, $C$. elegans exhibit gradual steering to orient in the preferred direction, a behavior called klinotaxis, as long as they are at an angle relative to the gradient that allows sensory sampling across head sweeps (lino and Yoshida, 2009; Kato et al., 2014). Steering and the biased random walk

Grant P40 OD010440.

We thank Xinyu Liu and Xianke Dong (McGill University) for providing photoresist masters for microfluidic devices. We also thank all members of the lab for helpful discussions and comments.

Correspondence should be addressed to Michael Hendricks at the above address, E-mail: michael.hendricks@mcgill.ca.

https://doi.org/10.1523/ENEURO.0121-18.2018

Copyright (C) 2018 Ouellette et al.

This is an open-access article distributed under the terms of the Creative Commons Attribution 4.0 International license, which permits unrestricted use, distribution and reproduction in any medium provided that the original work is properly attributed. operate on fundamentally different principles (Lockery, 2011). While the latter operates primarily by sensory integration over tens of seconds (Pierce-Shimomura et al., 1999; Luo et al., 2014a,b), steering requires constant, ongoing integration of sensory and motor information on a short time scale (lino and Yoshida, 2009; Kato et al., 2014).

We previously showed that each of a pair of unipolar interneurons called RIA can simultaneously encode both head movements and sensory inputs through two types of calcium signal (Hendricks et al., 2012). Local, compartmentalized calcium events in the ventral and dorsal segments of the RIA axon ( $\mathrm{nrV}$ and $\mathrm{nrD}$ ) were correlated with dorsal and ventral head bends, respectively (Fig. 1A,B). These axonal compartments correspond to sites of synaptic input from two pairs of head motor neurons, the SMDs (White and Southgate, 1986). We will refer to these compartmentalized events within the nerve ring as $\mathrm{mCa}^{2+}$, for motor-evoked calcium events. In addition to $\mathrm{mCa}^{2+}$ signals, removal of an attractive odor resulted in calcium increase throughout the entire axon, including nrV and $\mathrm{nrD}$, while odor presentation resulted in whole-axon suppression of calcium levels (Fig. 1C). These events will be referred to as $\mathrm{sCa}^{2+}$, for sensory-evoked calcium events. These responses are dependent on synaptic input from upstream sensory neurons and interneurons that synapse onto RIA exclusively in the "loop" region of the axon in the ventral nerve cord (Fig. 1A).

Within the nerve ring, $\mathrm{mCa}^{2+}$ and $\mathrm{sCa}^{2+}$ signals overlap spatially and temporally. We previously showed that these signals were genetically separable, and they were additive with one another within each nerve ring compartment. This additivity suggests a simple mechanism for integrating head orientation and sensory input to calculate the spatial distribution of a stimulus across each head sweep. We therefore proposed a model for steering based on asymmetric output from RIA to head motor neurons (Hendricks and Zhang, 2013). The basis of this model is that RIA mediates negative feedback onto head motor neurons, limiting the extent of head bending and setting gait amplitude. This is supported by the observation that impairing RIA function led to increased gait amplitude (Hendricks et al., 2012). When stimuli are absent or distributed symmetrically (Fig. $1 D$ ), this negative feedback is also symmetric. However, if stimulus changes are experienced across head bends, as when crawling orthogonal to a gradient, $\mathrm{sCa}^{2+}$ events are produced in phase with head bends, leading to enhanced negative feedback in the unfavored direction and suppressed negative feedback in the favored direction (Fig. 1E).

Here, we confirm and extend aspects of this model by examining simple head orientation behaviors and sensory encoding events in RIA, which have not been explored in detail and which reveal new features of sensorimotor integration in RIA. We propose a "gate and switch" model for RIA function in which sensory filtering prevents inappropriate activation of RIA when the head is not bent (gating), while $\mathrm{mCa}^{2+}$ asymmetry constitutes a switch that routes an inhibitory signal to dorsal or ventral motor neu- 


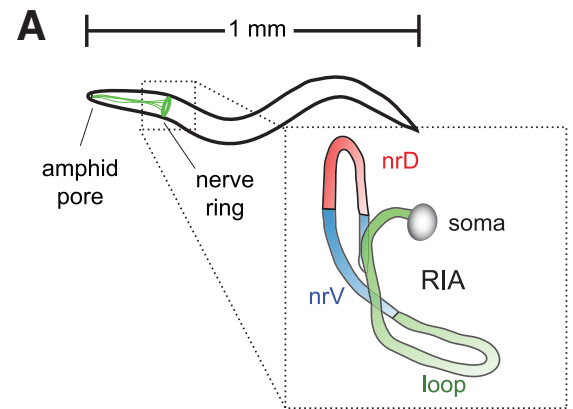

B

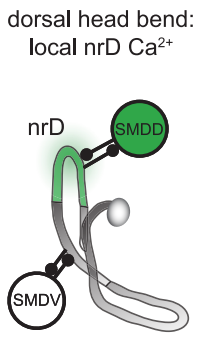

Motor encoding

ventral head bend: local nrV Caa ${ }^{2+}$

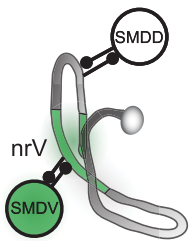

C

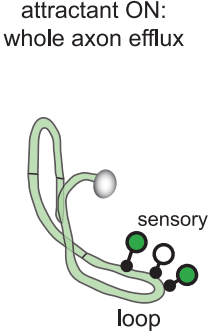

Sensory encoding

attractant OFF: whole axon influx

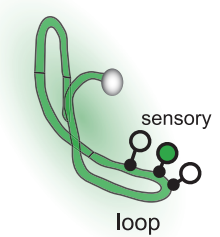

D

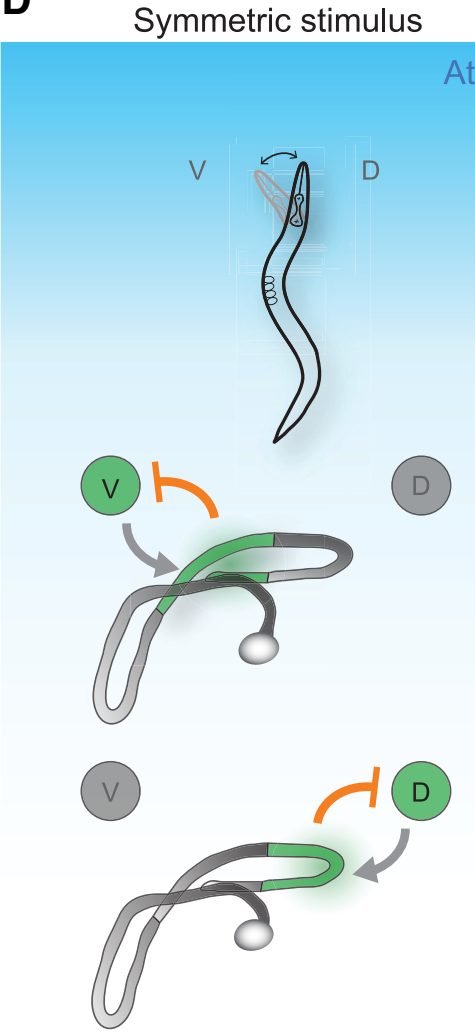

E

Asymmetric stimulus

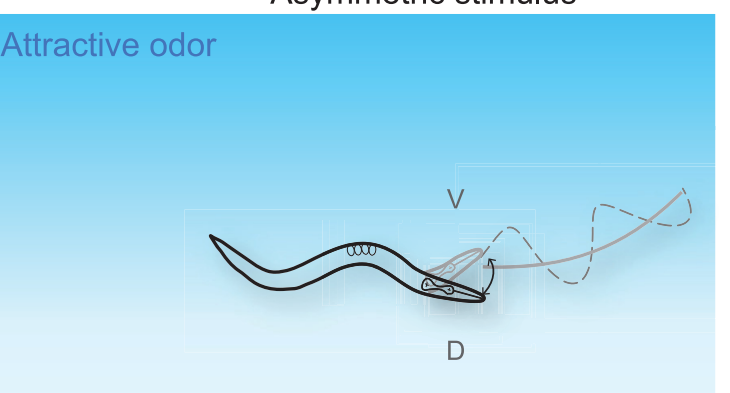

$\mathbf{F}$

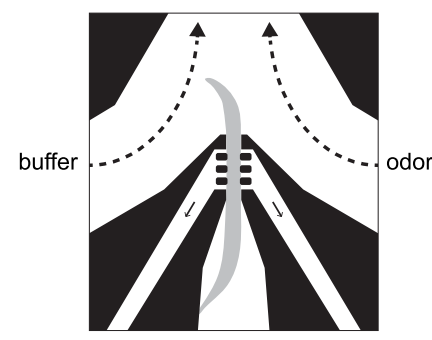

G

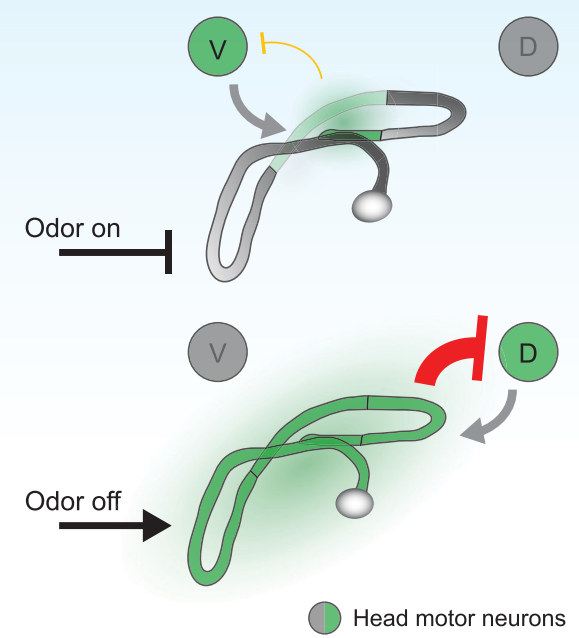

Figure 1. RIA overview and requirement for control of head orientation. $\boldsymbol{A}, \mathrm{RIA}$ is a unipolar interneuron whose axon extends into the ventral nerve cord and throughout the nerve ring. There are two RIAs, left and right, one is shown. $\boldsymbol{B}$, Two axonal compartments within the nerve ring, $\mathrm{nrV}$ and $\mathrm{nrD}$, encode head movements via local calcium signals $\left(\mathrm{mCa}^{2+}\right)$ triggered by muscarinic input from SMD head motor neurons. $\boldsymbol{C}$, Sensory pathways synapse on the loop domain of the RIA axon in the ventral nerve cord. Attractive stimuli lead to whole-axon reduction in calcium, while attractant removal causes whole axon calcium increases $\left(\mathrm{sCa}^{2+}\right)$. $\boldsymbol{D}$, Model for RIA function in steering behaviors. Where stimuli are symmetric across head bends, inhibitory output from RIA to motor neurons is symmetric. $\boldsymbol{E}$, If the stimulus changes across head sweeps, RIA output to motor neurons become asymmetric, driving steering behavior. $\boldsymbol{F}$, Schematic of head orientation measurement in response to a unilaterally presented stimulus. G, Estimation plot of difference between wild-type preference for odor as measured by percentage of the assay time $(120 \mathrm{~s})$ with their head within the odor stream and RIA::TeTx animals or animals treated with scopolamine. ANOVA $F_{(2,50)}=5.7771, p=0.0055$, $* p<0.05$, $* * p<0.01$ post hoc Student's $t$ test, $n=13, n=20, n=20$. V, ventral; $D$, Dorsal. Open circles and lines are mean \pm SD (top). Closed circles and lines are mean difference from control $\pm 95 \%$ confidence interval, shown with distribution of estimated means (see Materials and Methods).

rons to bias gait. Finally, we identify explanatory shortcomings of the model that require further study.

\section{Materials and Methods}

\section{Animals}

C. elegans were raised on nematode growth medium and fed with Escherichia coli strain OP50 according to standard methods (Brenner, 1974; Stiernagle, 2006). Experiments were conducted on young ( $3 \mathrm{~d}$ old) adult hermaphrodites. The strains used are N2, VC657 gar3(gk305)V, MMH099 yxls19 [Pglr-3a::GCaMP3.3, Punc122::DsRed], MMH100 yxls19; yxls20 [Pglr-3::TeTx: mCherry, Punc-122::GFP]; VC657 gar-3(gk305)V; ZC1792 gar-3(gk305)V; ZC1923 gar-3(gk305)V; yxls19; yxEx968 
A

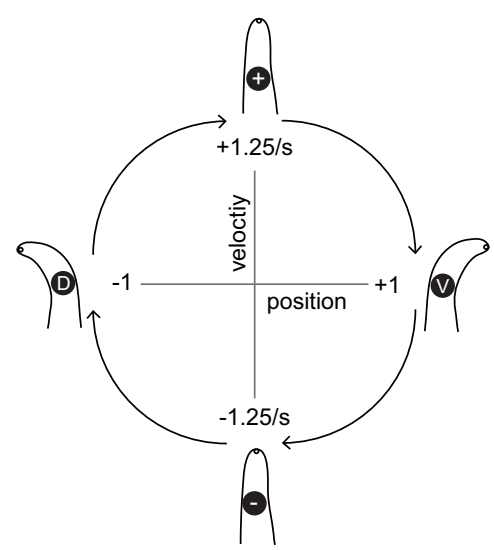

B

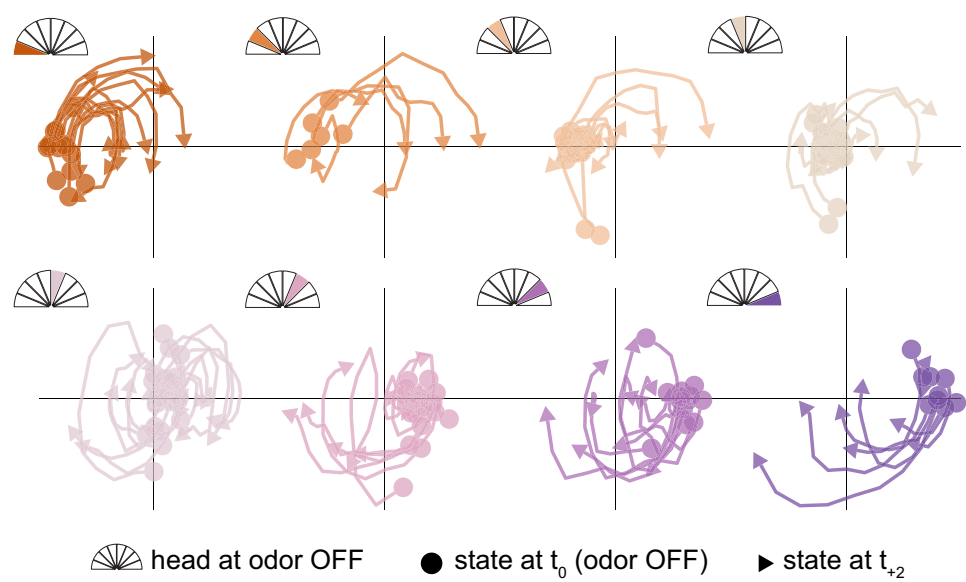

C

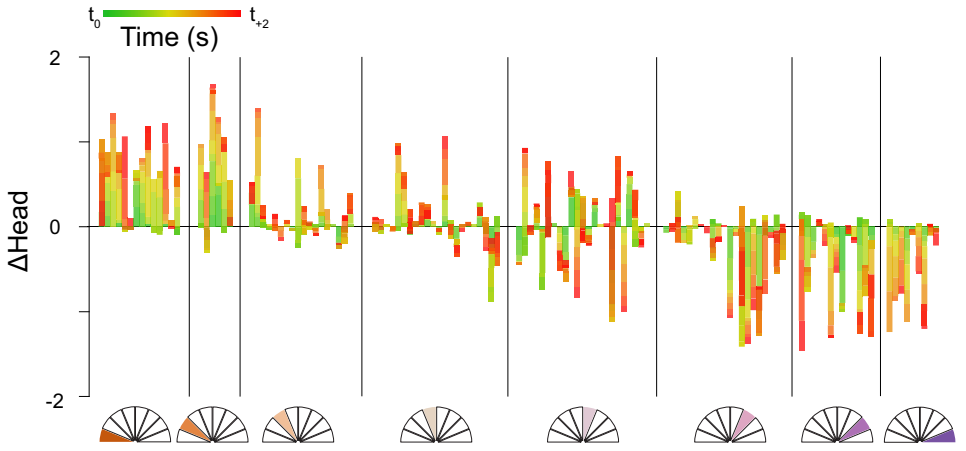

D

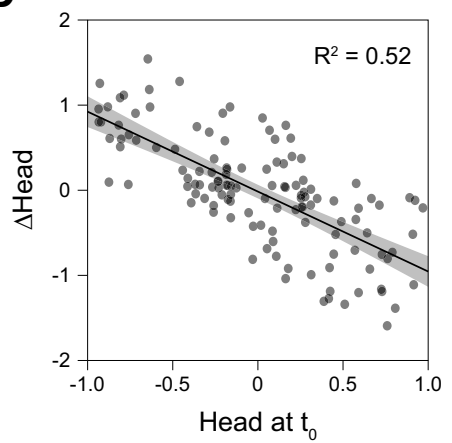

Figure 2. Responses to odor removal depend on posture. A, Schematic of position-velocity plots to illustrate oscillatory head movements. $\boldsymbol{B}$, Position-velocity trajectories from odor removal (circles) to $2 \mathrm{~s}$ after (arrowheads). $\boldsymbol{C}$, Head displacement in the $2 \mathrm{~s}$ immediately following odor removal, with the start position normalized to head orientation at the odor switch. $\boldsymbol{D}$, Relationship between head position at odor removal and the change in head position $2 \mathrm{~s}$ later. Linear regression $F_{(1,124)}=132.3145, p<0.0001 ; n=126$.

[Pglr-3::gar-3]. Molecular biology and strain generation was previously described (Hendricks et al., 2012). Pg/r-3:: TeTx:mCherry was generated by LR recombination of pSM-rfB-TeTx:mCherry destination and pCR8-Pglr-3 entry Gateway vectors described therein (Invitrogen), followed by injection at $25 \mathrm{ng} / \mu \mathrm{l}$. Tetanus toxin is expressed as a C-terminal fusion protein with mCherry. MMH099 is derived from the previously described strain ZC1508 after extensive outcrossing to N2. Some strains were provided by the Caenorhabditis Genetics Center (CGC).

\section{Microfluidic device fabrication}

Standard soft lithography methods were used to fabricate photoresist (SU8) masters for microfluidic devices (San-Miguel and Lu, 2013). Two-component polydimethylsiloxane (PDMS; Dow Corning Sylgard 184, Ellsworth Adhesives \#4019862) was mixed at 10:1 w/w, degassed under vacuum, poured over masters, degassed again, and cured at $60^{\circ} \mathrm{C}$ for at least $3 \mathrm{~h}$. Devices were replica mastered in a two-part epoxy resin (Smooth Cast 310, Sculpture Supply Canada \#796220) according to the manufacturer's instructions. Inlet holes were made with a Milltex $1 \mathrm{~mm}$ biopsy punch (Fisher). Chips were cleaned with ethanol and Scotch tape (3M) and then bonded to glass coverslips using air plasma generated by a handheld corona treater (Haubert et al., 2006; Electro-Technic Products). Coupling to fluid reservoirs was done by directly inserting PTFE microbore tubing (Cole-Parmer \#EW-06417-21) into inlet holes.

\section{Calcium imaging}

Fluorescence time lapse imaging (100-ms exposures, five frames per second) was performed as described (Hendricks et al., 2012). Briefly, animals were restrained in a microfluidic channel with a depth of $28 \mu \mathrm{m}$ to prevent movement in the $z$-axis and that allows the anterior portion of the head to move freely (Chronis et al., 2007). They were exposed to alternating streams of NGM buffer (1 mM $\mathrm{CaCl}_{2}, 1 \mathrm{mM} \mathrm{MgSO}_{4}$, and $25 \mathrm{mM} \mathrm{KPO}_{4}$; $\mathrm{pH}$ 6.0) or $100 \mu \mathrm{M}$ isoamyl alcohol (IAA; BioShop ISO900) in NGM buffer. Fluid flow was driven by negative pressure from a vacuum pump and controlled with a ValveBank (AutoMate Scientific). Images were captured at five frames per second, $100-m s$ exposures using a $40 \times$ objective. Movements in xy were corrected by stack registration, and ROIs corresponding to the RIA axonal domains were selected manually. GCaMP3 (Tian et al., 2009) intensity was measured from axonal compartments and normalized with the for- 
A

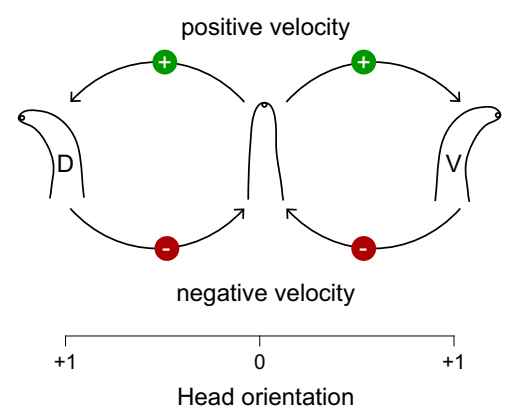

B

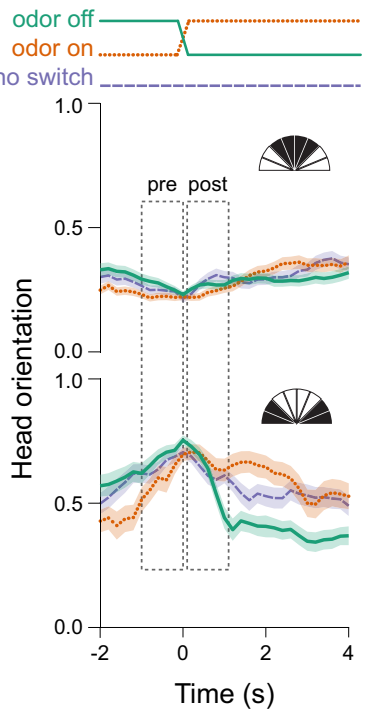

C
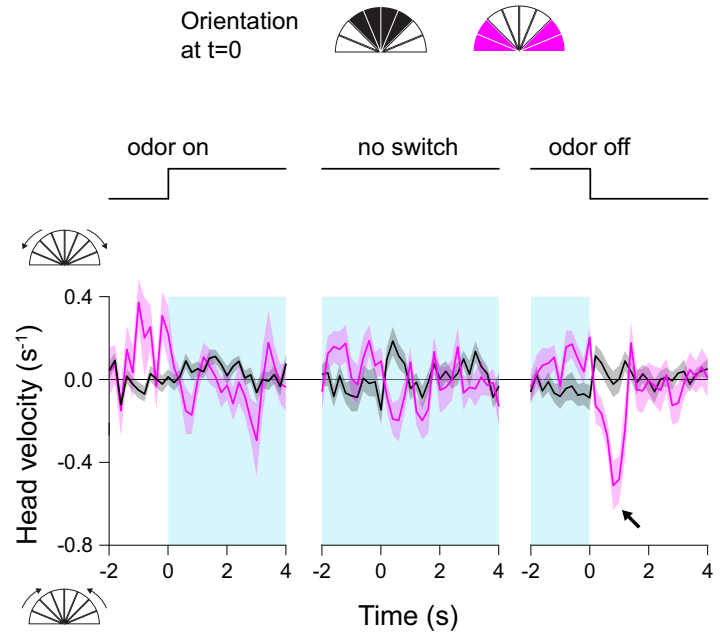

D
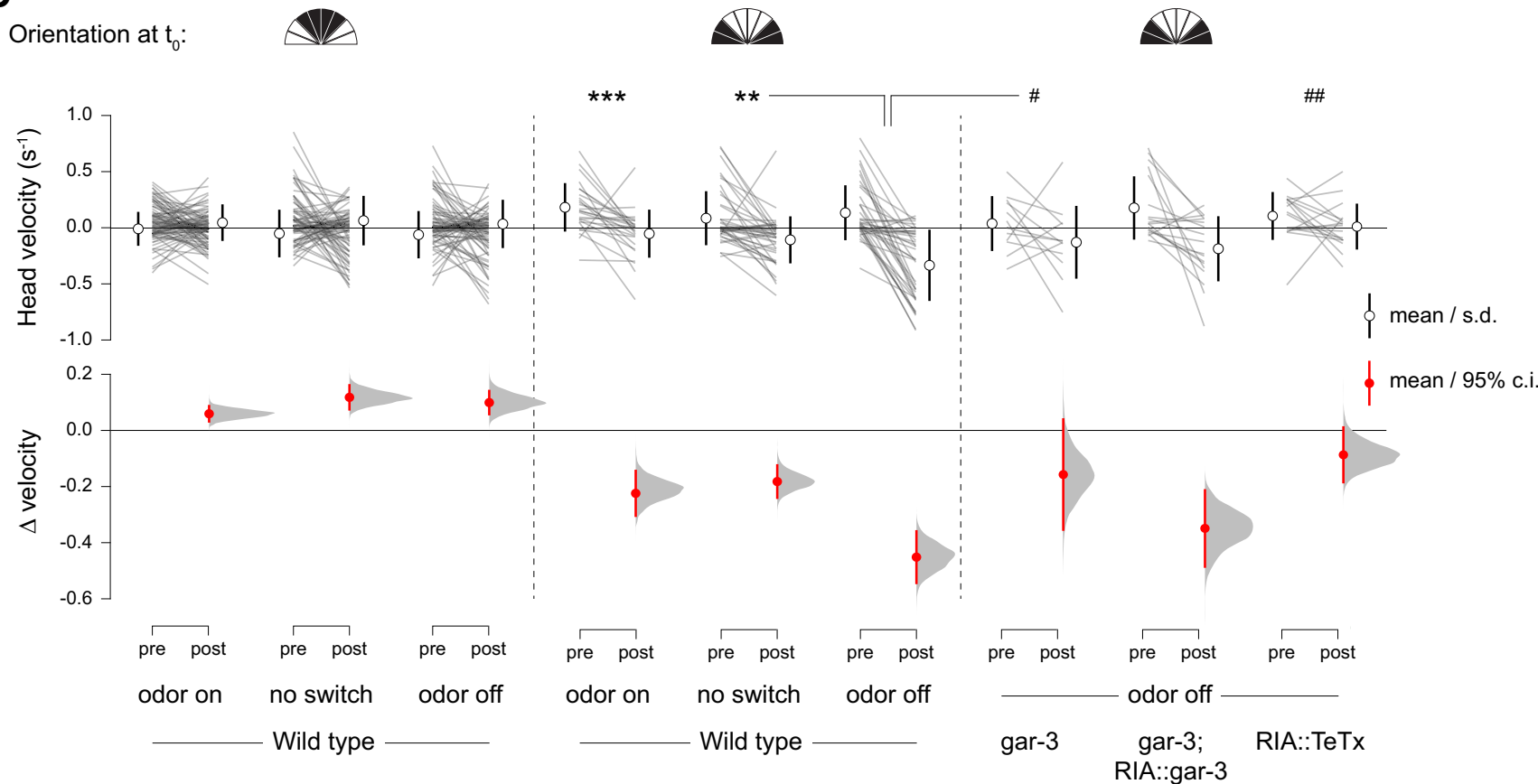

Figure 3. RIA mediates directional head withdrawal. A, To analyze head withdrawal behaviors, which are mirror-symmetric across the dorsoventral axis, head deflection in either the dorsal (D) or ventral (V) direction is defined as positive. Positive velocities correspond to bending away from the body axis and negative velocities indicate head withdrawal. $\boldsymbol{B}$, Mean plots of peristimulus head orientation at odor off, odor on, or in constant odor (no switch), binned according to whether the head is unbent (top) or bent in either direction (bottom) at the time of stimulus change $(t=0)$. Dashed "pre" and "post" boxes indicate time windows used for quantitation in $\boldsymbol{D}$. $\boldsymbol{C}$, Comparison of head velocity in response to stimulus changes (or no switch) when the head is bent or unbent. Arrow indicates characteristic head withdrawal in response to odor removal when the head is bent. $\boldsymbol{D}$, Paired mean pre- and post-switch head velocities (upper panels, open circles and lines are mean \pm SD) and estimations of the size of the head velocity change (lower panel, closed circles and lines are mean difference $\pm 95 \%$ confidence interval, along with probability distribution of means, see Materials and Methods). Odor switch (on, off, or constant odor) has no effect on head movements when the head is not bent (left panel, repeated measures ANOVA $\left.F_{(2,263)}=0.9386, p=0.3925, n=100, n=82, n=84\right)$. Sharp decreases in velocity are seen when odor removal occurs when the head is bent, but not for odor presentation or constant odor (middle panel, repeated measures ANOVA, $\left.F_{(2,109)}=7.7932, p=0.0007, n=26, n=44, n=42\right)$. In gar-3 mutants and animals expressing tetanus toxin in RIA these sharp decreases in head velocity are absent (right panel, repeated measures ANOVA $F_{(3,85)}=4.4105, p=0.0062, n=42, n=12, n=18$, $n=17$ ); $* * * p<0.001, * * p<0.01$ post hoc Student's $t$ test between odor conditions (on, off, no switch) for wild-type animals; \#\#p $<0.01$, \#p $<0.05$ post hoc Student's $t$ test between genetic manipulation (wild type, gar-3, gar-3 rescue, RIA::TeTx) for odor off responses during head bending. 
A

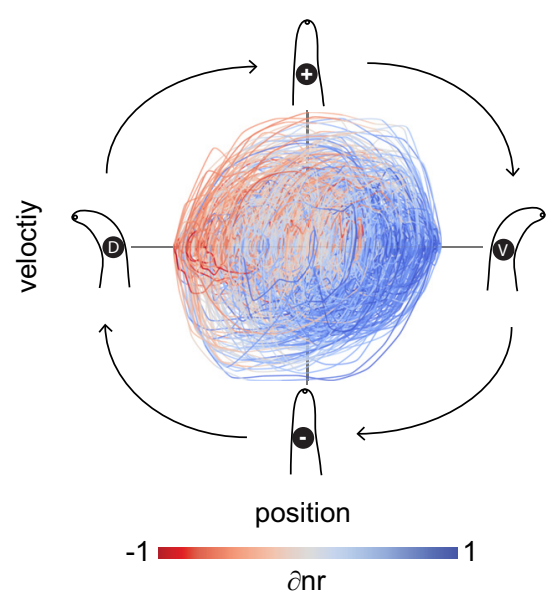

B

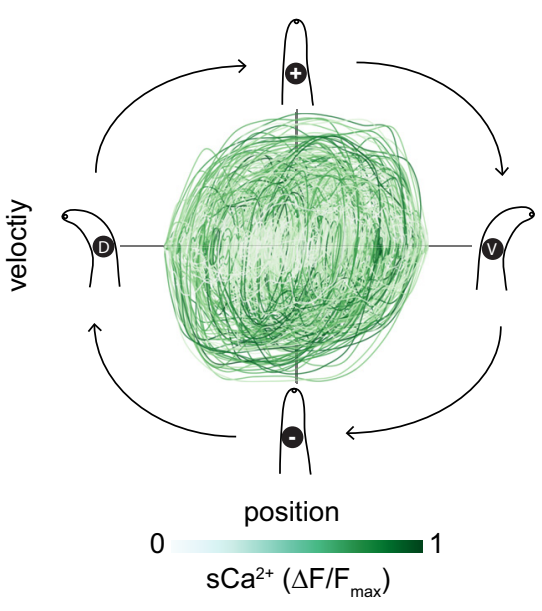

D

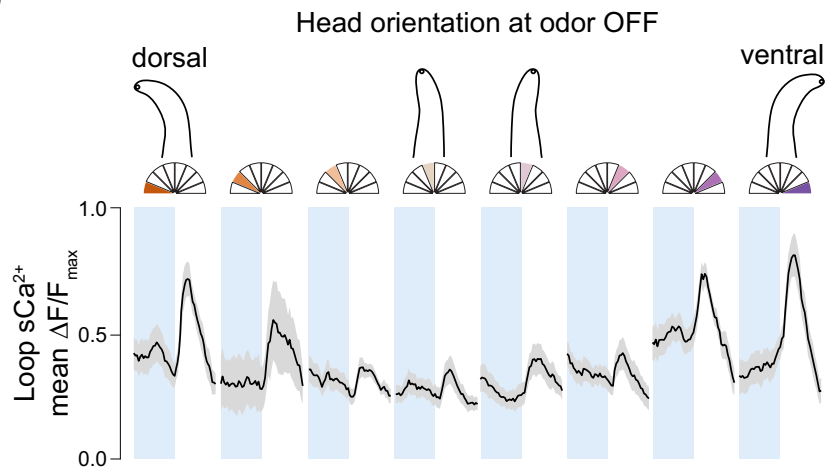

C

Head orientation

Loop sCa ${ }^{2+}$

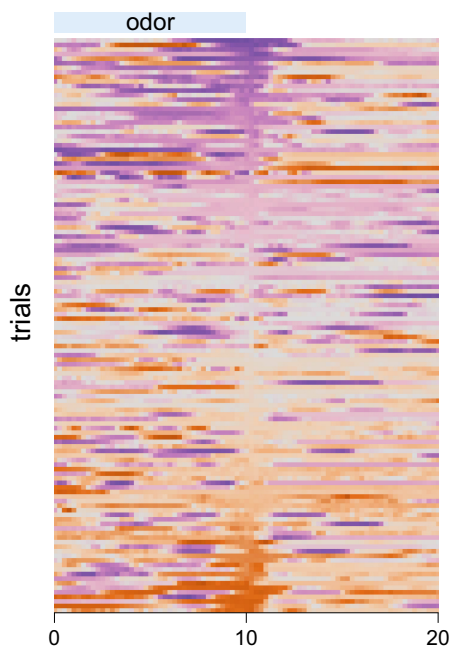

Time (s)
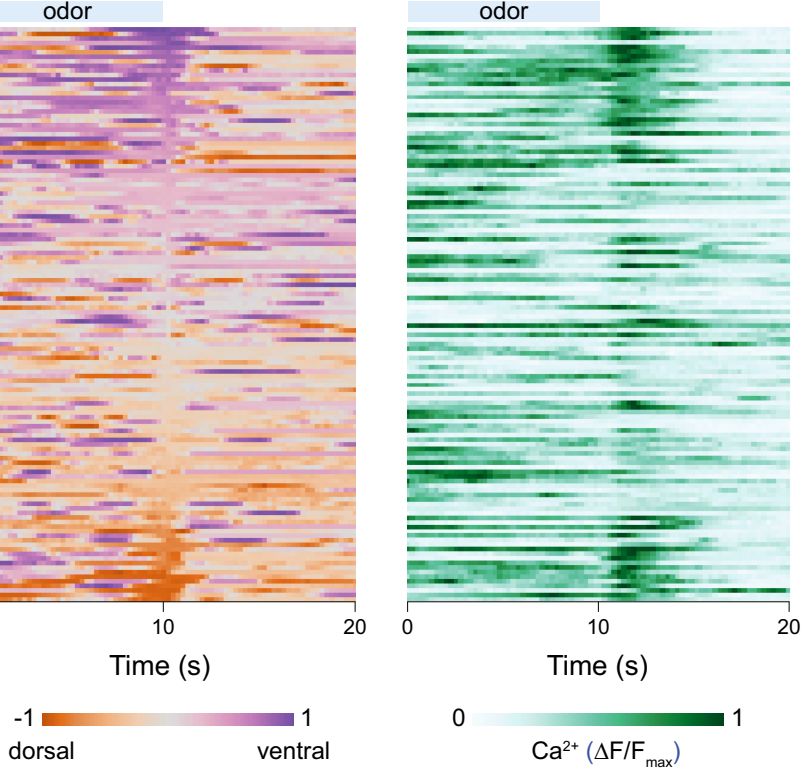

Time (s) $\mathrm{Ca}^{2+}\left(\Delta \mathrm{F} / \mathrm{F}_{\max }\right)$

E
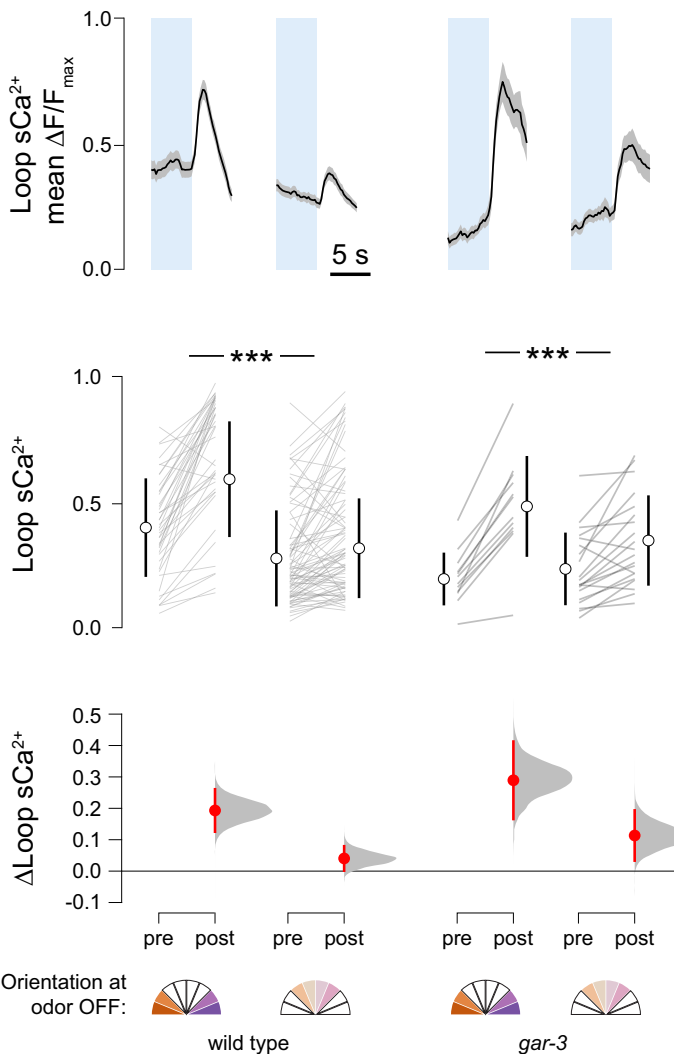

Figure 4. Head orientation gates sensory responses. $\boldsymbol{A}$, Head position-velocity trajectories color-coded by $\partial \mathrm{nr}(=\mathrm{nrV}-\mathrm{nrD})$, a measure of normalized $\mathrm{mCa}^{2+}$ asymmetry in $\mathrm{nrV}$ and $\mathrm{nrD}$. $\boldsymbol{B}$, Head position-velocity trajectories color-coded by loop calcium signal, $n=126,40$ s each. $\boldsymbol{C}$, Spontaneous head movements (left) and $\mathrm{sCa}^{2+}$ signals in the loop region of the RIA axon (right). Rows are matched and sorted according to head orientation at odor off $(t=10 \mathrm{~s})$. A linear regression of loop response magnitudes and head deflection in either direction was significant $\left(F_{(1,124)}=29.98, p<0.0001, n=126\right)$. $\boldsymbol{D}$. Mean traces of loop calcium responses $5 \mathrm{~s}$ before and after odor removal binned by head orientation at the odor off time point. Shading is SEM. Left to right, $n=14, n=6, n$ $=18, n=22, n=23, n=21, n=13, n=9$. $E$, Comparison of the magnitude of loop calcium responses to odor removal when the head is bent (head orientation $>0.5$ or $<-0.5$ ) or unbent (head orientation between -0.5 and 0.5 ). Pre- and post-measurements are 
continued

mean loop $\mathrm{Ca}^{2+}$ levels in $1 \mathrm{~s}$ windows just before odor OFF and centered on the peak of the mean response, respectively, shown as paired responses (lines) and mean \pm SD (open circles and lines). Estimations of the mean changes are shown below as mean difference $\pm 95 \%$ confidence interval (closed circles and lines) along with probability distributions of the means (see Materials and Methods). In both wild-type $(n=84, n=42)$ and gar-3 $(n=20, n=12)$ animals, the loop response magnitude is larger when the head is bent (repeated measures ANOVA $F_{(1,154)}=41.4529, p<0.0001, * * * p<0.001$ post hoc Student's $t$ test). gar-3 mutants have larger responses under both head orientation conditions, but there is no significant interaction between loop $\mathrm{Ca}^{2+}$ responses, head position at odor off, and genotype (repeated measures ANOVA $F_{(1,154)}=0.2145, p=0.6439$ ).

mula $\left(F_{t}-F_{\min }\right) /\left(F_{\max }-F_{\text {min }}\right)$. Asymmetry between the nerve ring compartments ( $\partial \mathrm{nr})$ was defined as (nrV - nrD). All calcium imaging analysis was done using the FIJI distribution of ImageJ (Schindelin et al., 2012). During calcium imaging, animals were free to move the anterior portion of their head, and these movements were captured as well. Motion artifacts affecting the GCaMP3 signal can in principle produce spurious correlations with the animal's movement. If our correlations were artifacts of animal movement, these correlations should persist in gar-3 mutants or in scopolamine-treated animals, in which there are no compartmentalized $\mathrm{mCa}^{2+}$ events. However, both in previous work using an identical imaging configuration (Hendricks, 2012) and here, correlations between head movement and local GCaMP3 signals are completely eliminated, demonstrating that they are not produced by motion artifacts.

\section{Head orientation assays}

Microfluidic chips (60 $\mu \mathrm{m}$ deep) were based on a design by McCormick et al. (2011). Fluid flow was gravity driven and controlled with manual one-way stopcocks (ColeParmer). Animals were exposed to streams of NGM buffer and $100 \mu \mathrm{M}$ IAA on either side of their heads. For scopolamine treatment, animals were immersed in NGM buffer containing $100 \mu \mathrm{M}$ scopolamine (Sigma) for $5 \mathrm{~min}$ before and during the assay. Worms were loaded individually into the chip, and once in place, their movements were recorded for $2 \mathrm{~min}$, with a switch between buffer and IAA at the 1-min mark, using a USB camera (Aven Mighty Scope, \#2700-200) and VirtualDub software.

\section{Head orientation measurements}

In head orientation assays, head bending was measured at each time point as the angle between the tip of the animal's nose and the midline of its body at the most anterior restrained point using MATLAB. For calcium imaging, head bending angle was calculated as the angle between the major axis of an ellipse fit to the free portion of the animal's head and neutral (unbent) position using FIJI. Comparison to previously used methods based on the ratio of pixels on either side of the midline (Hendricks et al., 2012) showed that these approaches yield equivalent results.

\section{Code accessibility}

MATLAB code used to measure head orientation in Figure 1 is available from the authors on request.

\section{Statistics and data presentation}

All time series data are presented as lines with shading indicating the standard error of the mean. Where compar- isons are made, we have adopted the use of estimation plots, which focus on effect sizes and confidence intervals (Gardner and Altman, 1986; Ho et al., 2018). Estimation plots employ a secondary axis that shows the size of the difference between test condition and control as a mean and $95 \%$ confidence interval. For paired comparisons, this is the within-group difference. A probability distribution of the test mean was produced by generating 2500 Bayesian bootstrapped means using sampling with replacement from the experimental data (Rubin, 1981). The implementation used here weighted each observed mean according to a gamma distribution with shape parameter $(n-1) / n$ and a scale parameter of 1 . Where multiple comparisons are made, significance markers are for post hoc Student's $t$ test with Bonferroni correction. All statistical analysis and plotting were done in JMP Pro 13 (SAS). Figures were prepared with Adobe Illustrator.

\section{Results}

\section{RIA and head orientation}

Ablating RIA does not grossly affect random walk dynamics in isotropic environments or in response to olfactory stimuli (Gray et al., 2005; Ha et al., 2010). In contrast to the temporal integration of the biased random walk, steering behavior depends on asymmetric head bends that propagate through the normal locomotor body wave, producing curved forward movement (lino and Yoshida, 2009; Izquierdo and Lockery, 2010; Lockery, 2011). To test the idea that RIA is involved in biasing head movements in response to an asymmetrically presented olfactory stimulus, we analyzed head movements in a microfluidic chip designed for this purpose (Fig. 1F). In this device, animals are restrained in approximately the middle third of their body using gentle suction. Two fluid streams flow on either side of the head. Animals exhibit spontaneous head bends and body undulations and are able to express a preference for one of the two streams by restricting their movements to keep their head in the preferred odor stream (McCormick et al., 2011).

Because our previous characterization of RIA physiology used the chemoattractant IAA (3-methylbutan-1-ol) and steering has been demonstrated in IAA gradients, we used it as an olfactory stimulus (Hendricks et al., 2012; Kato et al., 2014; Liu et al., 2018). We recorded each animal for two minutes. To control for any intrinsic bias in head bending direction, the odor and buffer streams were switched after one minute. Wild-type animals showed a clear preference for the IAA stream, as measured by the proportion of time spent with their heads bent toward the odor (Fig. 1G). 
A
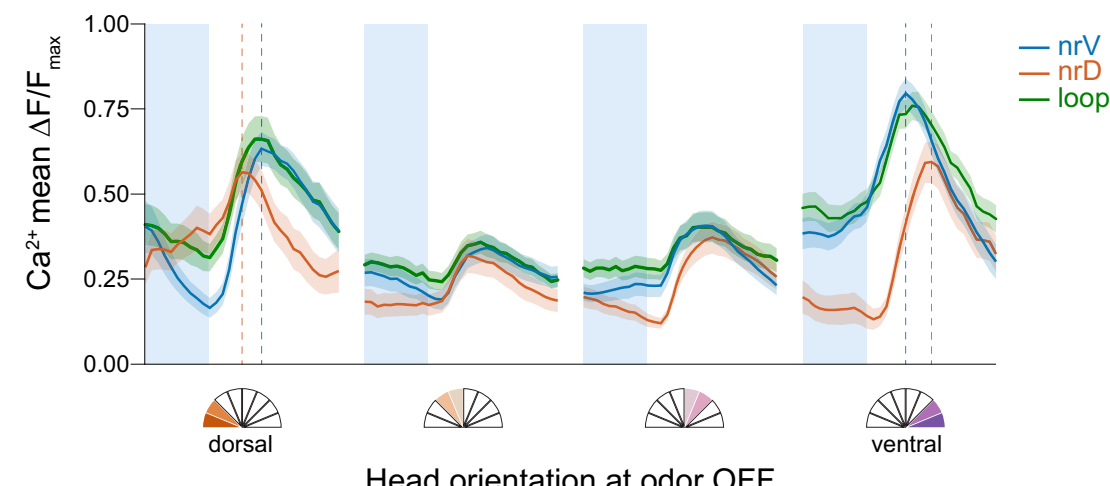

Head orientation at odor OFF

B

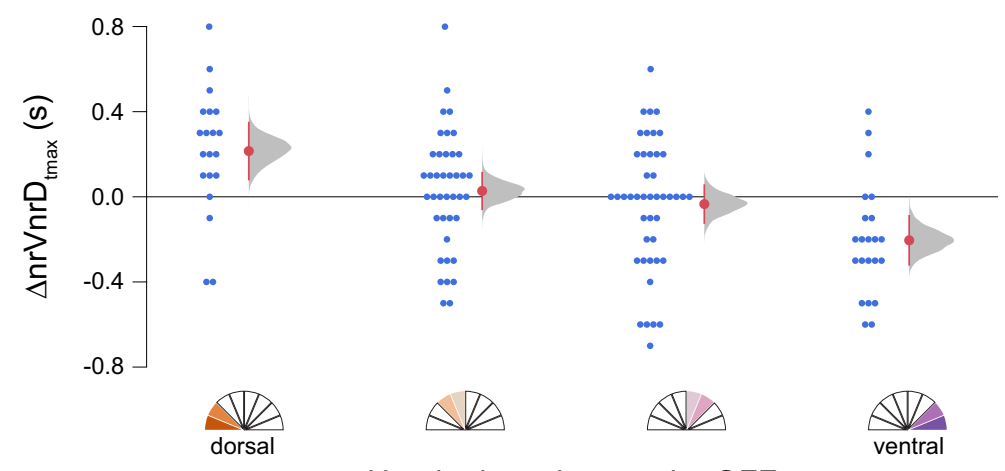

Head orientation at odor OFF

C
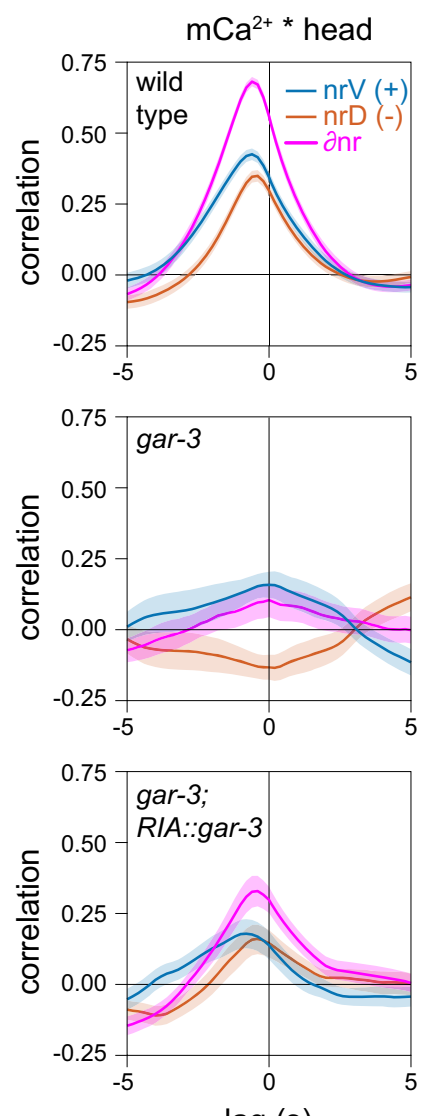

D

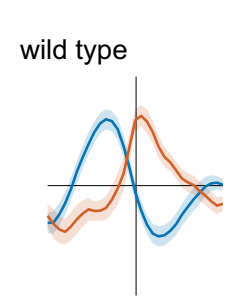

nrV, nrD * head velocity

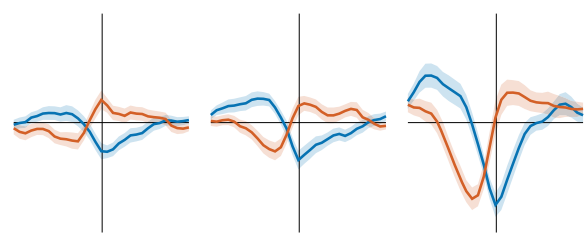

\begin{tabular}{ll} 
- $\operatorname{nrV}(+)$ & 0.25 \\
\hline $\operatorname{nrD}(-)$ & corr
\end{tabular}

gar-3
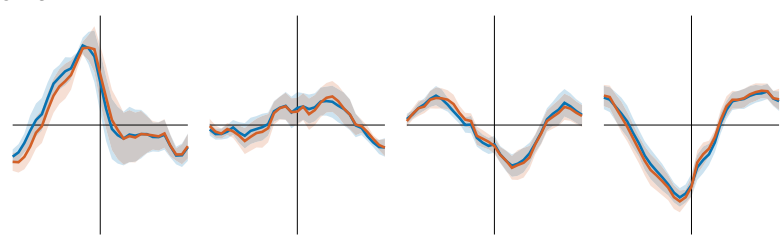

gar-3; RIA::gar-3

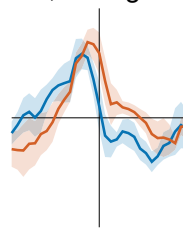

dorsal

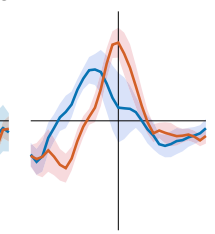

$\Delta D$

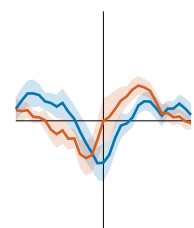

$\mathbb{A}$

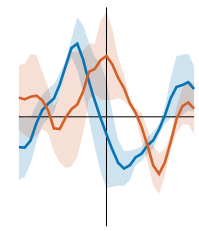

$\underset{\text { ventral }}{\mathbb{\bigotimes}}$

Head orientation at odor OFF

Figure 5. Temporal features of sensory responses in RIA axonal compartments. $\boldsymbol{A}$, Mean nrV, nrD, and loop responses on odor 
continued

removal. Shading is SEM, dashed lines indicate position of $\mathrm{nrV}$ and $\mathrm{nrD}$ mean peaks. $\boldsymbol{B}$, Head orientation predicts the mean lag between $\mathrm{nrV}$ and $\mathrm{nrD}$ peaks post-odor removal. ANOVA $F_{(1,124)}=13.47, p=0.0004$. $\boldsymbol{C}$, Cross-correlation of $\mathrm{nrV}$, $\mathrm{nrD}$, and $\mathrm{mCa}{ }^{2+}$ asymmetry ( $\partial \mathrm{nr}$ ) and head orientation, showing $\mathrm{mCa}^{2+}$ lag with head movements. gar-3 mutants lack mCa ${ }^{2+}$ and do not show head correlations. Re-expression of gar-3 cDNA in RIA partially rescues this relationship. $\boldsymbol{D}$, $\mathrm{nrV}$ and nrD cross-correlations with head velocity for wild-type, gar-3, and RIA-specific gar-3 rescue in a 6-s time window comprising 2-s pre-odor removal and 4-s post-odor removal in relation to head orientation at odor off. When the head was bent, we observed no lag between head withdrawal and peak calcium responses in the nerve ring compartment ipsilateral to the direction of bending. gar-3 mutants show no distinction between nerve ring compartments. Expression of gar-3 cDNA in RIA in gar-3 mutants does not rescue the temporal features of sensory responses in the nerve ring. Analysis groups are the same as Figure 4.

To test the role of RIA in this behavior, we used animals expressing tetanus toxin under an RIA-specific promoter. Tetanus toxin prevents synaptic vesicle release by cleaving synaptobrevin (Schiavo et al., 1992). In contrast to wild type, RIA::TeTx animals exhibited no preference (Fig. 1G). Next, we treated animals with scopolamine, a muscarinic acetylcholine receptor antagonist previously shown to abolish $\mathrm{mCa}^{2+}$ events (Hendricks et al., 2012). Like RIA:: TeTx animals, scopolamine treated animals show no directional preference (Fig. 1G). RIA-defective animals exhibit normal $\mathrm{sCa}^{2+}$ responses and RIA-ablated animals are capable of olfactory chemotaxis and thus are not sensory impaired (Ha et al., 2010; Hendricks et al., 2012). These results are consistent with the hypothesis that RIA is involved in responses to asymmetrically presented stimuli but not the temporal integration that underlies the biased random walk strategy.

\section{RIA mediates directional head withdrawal}

Steering requires posture-dependent responses to sensory inputs. We therefore examined the position and velocity of the head immediately before and after IAA removal in animals restrained in a chip that allows the head to move while facilitating temporal control of odor presentation and calcium imaging (Chronis et al., 2007; Hendricks et al., 2012). While analyzing head movements in restrained animals prevented us from measuring navigation behavior directly, it has the advantage of allowing
A

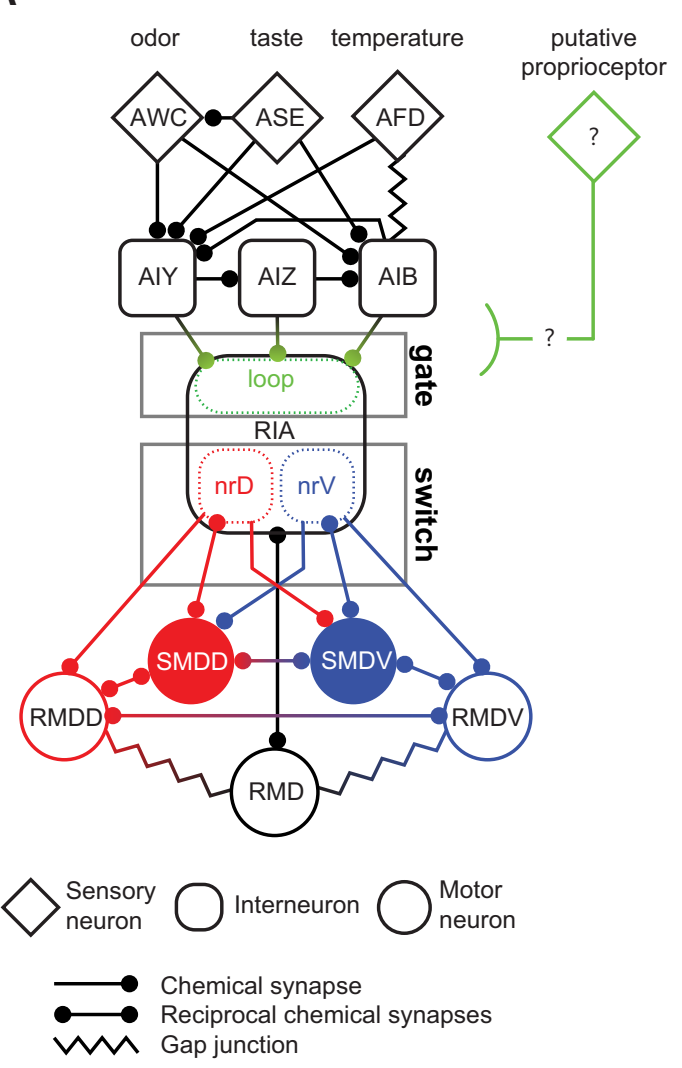

B

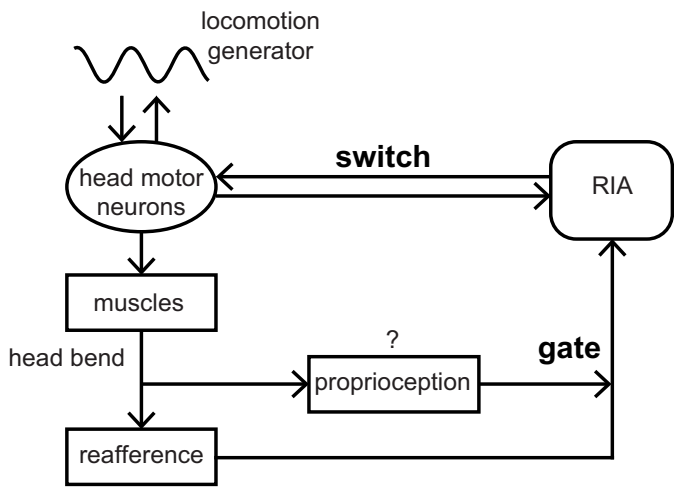

Figure 6. Gate and switch model for RIA function in head orientation behaviors. $\boldsymbol{A}, \mathrm{RIA}$ circuit diagram, with major upstream sensory pathways and head motor connections. Gating of sensory inputs to the loop domain and the nerve ring connections that constitute the dorsal-ventral switch. $\boldsymbol{B}$, Ethological model in which head movements simultaneously produce proprioception-gated reafference and efference copies that converge on RIA. RIA output to motor neurons biases the undulatory gait dorsally or ventrally when reafferent stimuli are asymmetric across head sweeps. 
us to unambiguously assign a stimulus change to a specific point in the head bending cycle.

We first examined whether there was any relationship between head movements and olfactory stimulus changes. To better visualize oscillatory head bending, we plotted head movements on a 2D position-velocity space that captures its phasic properties (Fig. 2A). We then analyzed head movements that occurred immediately after odor removal in relation to each phase of the head bending cycle (Fig. 2B). Large ventral head movements were associated with odor removal during dorsal head bends, and odor removal during ventral head bends triggered rapid dorsal head movements (Fig. 2C).

Because locomotion involves regular, oscillatory head movements, head withdrawal (returning toward center) is expected to follow immediately after each head bend. We predicted that stimulus-evoked movements would alter this normal oscillation. To distinguish the latter active head withdrawal events from normal behavioral progression through the oscillatory cycle, we compared head movements before and after odor withdrawal to head movements associated with either odor presentation or at an arbitrary time point with no stimulus change. Because responses to stimuli should be mirrored across the dorsoventral axis, for these experiments we defined head deflection in either direction as positive. Thus, positive velocities correspond to head bending and negative velocities to head withdrawal (Fig. 3A). While head bending does tend to be followed by head withdrawal (Fig. $3 B$ ), as expected, only odor removal elicits a characteristic, transient, rapid head movement and only when the head is bent (Fig. $3 C, D) \cdot \mathrm{mCa}^{2+}$ events rely on synaptic input from SMD motor neurons via the muscarinic AChR (mAChR) GAR- 3 (Hendricks et al., 2012). Our model suggests that these events play a role in modulating head movements in response to sensory stimuli. We therefore examined stimulus-evoked head withdrawal in gar-3 mutants and in RIA::TeTx animals. Neither of these strains exhibited stimulus-evoked head withdrawals (Fig. 3D). Expressing a wild-type gar-3 cDNA specifically in RIA showed partial rescue of odor removal-evoked head withdrawal, but these responses were not significantly different from those of gar-3 mutants (Fig. 3D).

\section{RIA sensory responses are gated according to head position}

Models of steering rely on symmetry breaking by phasic modulation of the effects of sensory input on motor networks (Lockery, 2011). Support for a role for RIA in this process was provided in a recent paper in which selective inactivation of RIA synapses in either $\mathrm{nrV}$ or $\mathrm{nrD}$ produced curved locomotion in the predicted direction (Liu et al., 2018). This relies on generating deeper head bends in the direction of curvature, essentially the inverse of head withdrawal. Both mechanisms rest on the idea that differential $\mathrm{mCa}^{2+}$ levels in $\mathrm{nrV}$ and $\mathrm{nrD}$ function as a switch to route output from RIA to ventral or dorsal motor neurons. This output is predicted to be inhibitory and to limit head bends in the unfavorable direction. However, restricting head bends must be balanced against the need to main- tain forward locomotion. Because $\mathrm{mCa}^{2+}$ levels are only partially correlated with head movements and frequently do not show dorsal-ventral differences (Hendricks et al., 2012), there is a potential for inappropriate symmetrical activation or inhibition, which would be predicted to disrupt the animal's gait.

We therefore analyzed the relationship between head movements, $\mathrm{mCa}^{2+}$ asymmetry in the nerve ring, and $\mathrm{sCa}^{2+}$ responses in the loop domain. Consistent with previous observations, $\mathrm{mCa}^{2+}$ asymmetry (2nr) shows a clear relationship to head oscillations (Fig. 4A). There is no apparent overall relationship between loop $\mathrm{sCa}^{2+}$ and head movement (Fig. 4B). Because odor switches occur at set time points while head movements are spontaneous, the relationship between head position and odor removal is arbitrary. Spontaneous $\mathrm{sCa}^{2+}$ events may mask a relationship between head movement and stimulus-evoked events because the latter are much less common. We therefore examined whether stimulusevoked loop $\mathrm{sCa}^{2+}$ events that occur on odor removal are dependent on head phase.

We found an unexpected relationship between loop responses and head position at the time of odor removal. Odor removal triggers calcium influx throughout the RIA axon via inputs from upstream interneurons, and the magnitude of these responses was large when the animal's head was bent, in either direction, and small or absent when it was not (Fig. 4C,D). This effect is the same for dorsal or ventral bends, so as in Figure 3, we defined all bends as positive and classified head positions as "bent" or "not bent" if they were more or less than half the maximal degree of head deflection, respectively. This posture classification revealed a robust difference in the magnitude of $\mathrm{sCa}^{2+}$ responses in response to odor removal (Fig. 4E).

Posture dependence in behavioral responses to pulsed thermal stimuli was observed by Stephens et al. (2008). In that study, animal posture was defined through a dimensional reduction procedure, and an animal's state defined by the first two components was predictive of responses to a sudden temperature increase. With only a behavioral readout, it is difficult to tell whether posture dependence is based on an active neural mechanism or is a constraint of the motor program. In the case of RIA, we have clear evidence of a sensory gating mechanism. This has important implications for sensory coding in RIA. First, gating allows preferential attention to stimuli encountered during head bends, consistent with a function in steering in response to asymmetrically distributed stimuli. Second, it resolves the issue raised above of potential gait disruption caused by symmetrical output from RIA to head motor neurons. Gating ensures that large $\mathrm{sCa}^{2+}$ events are only likely to occur when $\mathrm{mCa}^{2+}$ in the nerve ring is asymmetric, i.e., only when the downstream "switch" is engaged to favor either dorsal or ventral synaptic output.

The sensory gating mechanism may act directly on RIA or be a feature of upstream interneurons. We first tested the hypothesis that the gate and switch mechanisms are in fact the same: motor inputs via GAR-3 that produce local $\mathrm{mCa}^{2+}$ events may also somehow gate $\mathrm{sCa}^{2+}$ responses. To do so we repeated our analysis of sensory- 
evoked $\mathrm{sCa}^{2+}$ relative to head orientation in gar-3 mutants. In this mutant, $\mathrm{mCa}^{2+}$ events are absent while $\mathrm{sCa}^{2+}$ responses are intact, leading to perfectly symmetrical calcium activity in the nerve ring (Hendricks et al., 2012). gar-3 mutants exhibited normal sensory gating (Fig. 4E). This suggests that an independent gating mechanism exists at the level of the RIA loop or upstream interneurons. There are several candidate mechanoreceptor neurons in the head, and this mechanism may rely on a hitherto unidentified proprioceptive pathway.

\section{Head bending desynchronizes local calcium responses to sensory input}

How $\mathrm{sCa}^{2+}$ and $\mathrm{mCa}^{2+}$ interact and influence synaptic release is critical for understanding of RIA function. We previously showed that $\mathrm{sCa}^{2+}$ and $\mathrm{mCa}^{2+}$ are additive within the nerve ring compartments (Hendricks et al., 2012). Therefore, we analyzed $n r V$ and $n r D$ responses separately during whole-axon $\mathrm{sCa}^{2+}$ events with respect to each other and head velocity.

We found that head bending introduced a notable lag in peak responses within the nerve ring that was dependent on head orientation (Fig. $5 A, B$ ). The polarity of this lag depended on head position, such that the ipsilateral nerve ring compartment exhibited a shorter rise time and earlier peak with respect to the contralateral compartment. This is consistent with a simple additive model of $\mathrm{mCa}^{2+}$ and $\mathrm{sCa}^{2+}$. That is, the nerve ring compartment corresponding to the direction of head bending exhibits not only a stronger calcium signal but a shorter latency to peak calcium levels.

As previously shown, we observed that $\mathrm{mCa}^{2+}$ responses typically lag behind head movements (Fig. 5C). Possible reasons for this lag include the relative timing of the muscle contractions (via nicotinic acetylcholine receptors) and $\mathrm{mCa}^{2+}$ (via muscarinic receptors) elicited by acetylcholine released from head motor neurons. We have interpreted this lag as evidence that $\mathrm{mCa}^{2+}$ in RIA function as a representation of ongoing head movement. To understand how the shift in peak local calcium (the sum of $\mathrm{sCa}^{2+}$ and $\mathrm{mCa}^{2+}$ calcium sources within each compartment) might relate to stimulus-evoked head withdrawal, we examined the relative timing of these events. We performed cross-correlation analysis of head velocities with respect to $\mathrm{nrV}$ and $\mathrm{nrD}$ calcium dynamics in a restricted time window corresponding to odor removal. In these plots, positive peaks near the $y$-axis indicate a calcium signal that is coincident with head motion. Those to the left are calcium signals that are delayed relative to head motion, and thus are unlikely to play a causal role in the initiation of the behavior.

During sensory stimulation (odor removal) while the head is bent, peak sensory responses in the nerve ring are coincident with head withdrawal in the contralateral direction but lagged in the ipsilateral direction (Fig. 5D). That is, when the head is bent ventrally, the peak nrV calcium signal is synchronous with the highest head velocity in the dorsal direction (withdrawal) while nrD calcium lags, and vice versa during dorsal head bends. When the head is not bent, this relationship is lost due to the absence of strong $\mathrm{sCa}^{2+}$ responses. This desynchronization is ab- sent in gar-3 mutants, which completely lack $\mathrm{mCa}^{2+}$ (Hendricks et al., 2012). Re-expression of gar-3 specifically in RIA restored some compartmentalization (Fig. 5D) but was not sufficient to rescue the temporal structure of stimulus-evoked responses in $\mathrm{nrV}$ and $\mathrm{nrD}$, which may relate to its failure to fully rescue head withdrawal (Fig. 4D). This implies either GAR-3 plays additional roles outside RIA in mediating the temporal features of RIA signaling and head withdrawal, or that the rescue construct causes mislocalization, inappropriate expression levels, or otherwise does not sufficiently restore GAR-3 function in RIA.

Overall, these results show that the additive properties of $\mathrm{mCa}^{2+}$ and $\mathrm{sCa}^{2+}$ lead to decoupling of local calcium event timing in the nerve ring in response to odor removal. This has important functional implications for $\mathrm{mCa}^{2+}$. Because these signals slightly lag head movement, it was unclear how they might play an instructive role in controlling rapid response to sensory stimulus. Our results suggest that one possible role is to function additively with $\mathrm{sCa}^{2+}$ to reduce latency and facilitate more rapid transmitter release on one side of the RIA axon than the other, triggering head withdrawal.

\section{Discussion}

RIA is uniquely well-situated in the $C$. elegans wiring diagram to play a role in integrating head orientation and sensory input. It is one of very few interneurons that receive substantial motor feedback, and it receives convergent input from odor, taste, and temperature sensory pathways. Here, we confirmed a role for RIA in head orientation in response to an asymmetrically presented stimulus, following previous work demonstrating roles for RIA in steering in response to odor and temperature (Mori and Ohshima, 1995; Liu et al., 2018).

The recent study by Liu et al. (2018) demonstrated that asymmetrically suppressing neurotransmitter release from RIA, mimicking an attractive odor being presented on that side, led to deeper head bends and curving in that direction. Here, we describe the mirror-image behavior, head withdrawal and limiting of head bends in response to odor removal. Both are predicted by our model to contribute to steering (Fig. 1). Blocking RIA synaptic release or genetically removing asymmetric local $\mathrm{mCa}^{2+}$ in the RIA axon prevented directional head withdrawal. We identified a posture-dependent sensory gating mechanism that may prevent aberrant symmetric RIA output and causes RIA to be selectively responsive to stimuli encountered during head bending.

Phasic behaviors that produce reafferent stimulation often exhibit sensory gating coupled to the behavior. In mice, examples include olfaction, where cholinergic inputs to the olfactory bulb modulate sensory gain in phase with respiration during sniffing, and a functionally similar gating mechanism is evident during whisking (Eggermann et al., 2014; Rothermel et al., 2014). RIA may be part of a similar circuit mechanism, in which phasic inputs from head motor networks both gate sensory responses and tune motor outputs to respond appropriately to spatially asymmetric stimuli. 
A shortcoming of our model is that the rapid head withdrawal movements we observed, which often involve sudden reversals of head velocity, probably cannot be explained by inhibition of ipsilateral motor neurons alone but must also involve contralateral motor neuron activation and muscle contraction. In the nerve ring, the RIA axon receives input from ventral head motor neurons only in $\mathrm{nrV}$ and dorsal motor neurons only in $\mathrm{nrD}$. However, output synapses from RIA to SMD and RMD head motor neurons are not segregated in the same way. nrV and nrD both contain interspersed output synapses to both dorsal and ventral motor neurons. However, the postsynaptic anatomic arrangement of these synapses suggest that these connections may be of opposite valence (Fig. 6A). Ipsilateral connections are in the distal portion of the axon near neuromuscular junctions, while contralateral synapses are clustered near the motor neuron cell body. This is at least consistent with the possibility of differing effects of output from $\mathrm{nrV}$ and $\mathrm{nrD}$ on their ipsilateral contralateral motor neuron targets. Exploration of this will require identification of postsynaptic receptors and direct measurement of RIA's effects on its target neurons.

Our observation of dynamic sensory gating adds a new layer to the RIA sensorimotor circuit and resolves potential shortcomings of our previous model, in which RIA outputs but not sensory representations are regulated by head movements. We propose a two-level gate and switch model, in which an unidentified posture-dependent signal gates sensory $\mathrm{sCa}^{2+}$ responses while differential $\mathrm{mCa}^{2+}$ between the nerve ring compartments functions as a switch to produce asymmetric synaptic output from the $\mathrm{nrV}$ and $\mathrm{nrD}$ axonal domains (Fig. $6 A, B$ ). This switch may depend on the temporal decoupling we observed between $\mathrm{nrV}$ and $\mathrm{nrD}$ during sensory-evoked responses when the head is bent. $\mathrm{mCa}^{2+}$ introduces a temporal bias that reduces the time to peak response in the compartment contralateral to the direction of head withdrawal. This peak is coincident with head withdrawal, and the duration of the behavior is similar to the lag introduced between the two axonal compartments. Again, how plausible a mechanism this is will depend on analyzing the effects of these calcium events on synaptic release from RIA and the activity of postsynaptic motor neurons.

Despite its small size and simple morphology, RIA exhibits striking computational potential as a sensorimotor integrator. Along with clear behavioral readouts, this relative simplicity makes it an attractive system to probe sensorimotor integration at the cellular level, both in terms of molecular mechanisms and circuit logic. This work underscores the fundamental role of circuits that link self-motion and ongoing behavior to interpretation of the sensory environment.

\section{References}

Berg HC (1975) Bacterial behaviour. Nature 254:389-392. Medline Brenner S (1974) The genetic of Caenorhabditis elegans. Genetics 77:71-94. Medline

Calhoun AJ, Murthy M (2017) Quantifying behavior to solve sensorimotor transformations: advances from worms and flies. Curr Opin Neurobiol 46:90-98. CrossRef Medline
Chronis N, Zimmer M, Bargmann Cl (2007) Microfluidics for in vivo imaging of neuronal and behavioral activity in Caenorhabditis elegans. Nat Methods 4:727-731. CrossRef Medline

Crapse TB, Sommer MA (2008) Corollary discharge across the animal kingdom. Nat Rev Neurosci 9:587-600. CrossRef Medline

Eggermann E, Kremer Y, Crochet S, Petersen CCH (2014) Cholinergic signals in mouse barrel cortex during active whisker sensing. Cell Rep 9:1654-1660. CrossRef Medline

Gardner MJ, Altman DG (1986) Confidence intervals rather than P values: estimation rather than hypothesis testing. Br Med J 292: 746-750. Medline

Gray JM, Hill JJ, Bargmann Cl (2005) A circuit for navigation in Caenorhabditis elegans. Proc Natl Acad Sci USA 102:3184-3191. CrossRef Medline

Ha HI, Hendricks M, Shen Y, Gabel CV, Fang-Yen C, Qin Y, ColónRamos D, Shen K, Samuel ADT, Zhang Y (2010) Functional organization of a neural network for aversive olfactory learning in Caenorhabditis elegans. Neuron 68:1173-1186. CrossRef

Haubert K, Drier T, Beebe D (2006) PDMS bonding by means of a portable, low-cost corona system. Lab Chip 6:1548-1549. CrossRef Medline

Hedgecock EM, Russell RL (1975) Normal and mutant thermotaxis in the nematode Caenorhabditis elegans. Proc Natl Acad Sci USA 72:4061-4065. Medline

Hendricks M, Ha H, Maffey N, Zhang Y (2012) Compartmentalized calcium dynamics in a $C$. elegans interneuron encode head movement. Nature 487:99-103. CrossRef

Hendricks M, Zhang Y (2013) Complex RIA calcium dynamics and its function in navigational behavior. Worm 2:e25546. CrossRef Medline

Ho J, Tumkaya T, Aryal S, Choi H, Claridge-Chang A (2018) Moving beyond $\mathrm{P}$ values: Everyday data analysis with estimation plots. bioRxiv. Advance online publication. Retrieved from July 26, 2018. doi: $10.1101 / 377978$.

lino Y, Yoshida K (2009) Parallel use of two behavioral mechanisms for chemotaxis in Caenorhabditis elegans. J Neurosci 29:53705380. CrossRef Medline

Izquierdo EJ, Lockery SR (2010) Evolution and analysis of minimal neural circuits for klinotaxis in Caenorhabditis elegans. J Neurosci 30:12908-12917. CrossRef Medline

Kato S, Xu Y, Cho CE, Abbott LF, Bargmann Cl (2014) Temporal responses of $C$. elegans chemosensory neurons are preserved in behavioral dynamics. Neuron 81:616-628. CrossRef Medline

Liu H, Yang W, Wu T, Duan F, Soucy E, Jin X, Zhang Y (2018) Cholinergic sensorimotor integration regulates olfactory steering. Neuron 97:390-405.e3. CrossRef

Lockery SR (2011) The computational worm: spatial orientation and its neuronal basis in C. elegans. Curr Opin Neurobiol 21:782-790. CrossRef Medline

Luo L, Cook N, Venkatachalam V, Martinez-Velazquez LA, Zhang X, Calvo AC, Hawk J, Maclnnis BL, Frank M, Ng JHR, Klein M, Gershow M, Hammarlund M, Goodman MB, Colón-Ramos DA, Zhang Y, Samuel ADT (2014a) Bidirectional thermotaxis in Caenorhabditis elegans is mediated by distinct sensorimotor strategies driven by the AFD thermosensory neurons. Proc Natl Acad Sci USA 111:2776-2781. CrossRef

Luo L, Wen Q, Ren J, Hendricks M, Gershow M, Qin Y, Greenwood J, Soucy ER, Klein M, Smith-Parker HK, Calvo AC, Colón-Ramos DA, Samuel ADT, Zhang Y (2014b) Dynamic encoding of perception, memory, and movement in a $C$. elegans chemotaxis circuit. Neuron 82:1115-1128. CrossRef

McCormick KE, Gaertner BE, Sottile M, Phillips PC, Lockery SR (2011) Microfluidic devices for analysis of spatial orientation behaviors in semi-restrained Caenorhabditis elegans. PLoS One 6:e25710. CrossRef Medline

Mori I, Ohshima Y (1995) Neural regulation of thermotaxis in Caenorhabditis elegans. Nature 376:344-348. CrossRef Medline

Pierce-Shimomura JT, Morse TM, Lockery SR (1999) The fundamental role of pirouettes in Caenorhabditis elegans chemotaxis. J Neurosci 19:9557-9569. Medline 
Rothermel M, Carey RM, Puche A, Shipley MT, Wachowiak M (2014) Cholinergic inputs from Basal forebrain add an excitatory bias to odor coding in the olfactory bulb. J Neurosci 34:4654-4664. CrossRef Medline

San-Miguel A, Lu H (2013) Microfluidics as a tool for C. elegans research. WormBook. Advance online publication. Retrieved from September 24, 2013. doi: 10.1895/wormbook.1.162.1.

Schiavo G, Benfenati F, Poulain B, Rossetto O, Polverino De Laureto P, DasGupta BR, Montecucco C (1992) Tetanus and botulinum-B neurotoxins block neurotransmitter release by proteolytic cleavage of synaptobrevin. Nature 359:832-835. CrossRef Medline

Schindelin J, Arganda-Carreras I, Frise E, Kaynig V, Longair M, Pietzsch T, Preibisch S, Rueden C, Saalfeld S, Schmid B, Tinevez J-Y, White DJ, Hartenstein V, Eliceiri K, Tomancak P, Cardona A (2012) Fiji: an open-source platform for biological-image analysis. Nat Methods 9:676-682. CrossRef Medline
Stephens GJ, Johnson-Kerner B, Bialek W, Ryu WS (2008) Dimensionality and dynamics in the behavior of $C$. elegans. PLoS Comput Biol 4:e1000028. CrossRef Medline

Stiernagle T (2006) Maintenance of C. elegans. WormBook. Advance online publication. Retrieved from February 11, 2006. doi: 10.1895/wormbook.1.101.1.

Straka H, Simmers J, Chagnaud BP (2018) A new perspective on predictive motor signaling. Curr Biol 28:R232-R243. CrossRef Medline

Tian L, Hires SA, Mao T, Huber D, Chiappe ME, Chalasani SH, Petreanu L, Akerboom J, McKinney SA, Schreiter ER, Bargmann Cl, Jayaraman V, Svoboda K, Looger LL (2009) Imaging neural activity in worms, flies and mice with improved GCaMP calcium indicators. Nat Methods 6:875-881. CrossRef Medline

White JG, Southgate E (1986) The structure of the nervous system of the nematode Caenorhabditis elegans. Philos Trans R Soc Lond B Biol Sci 314:1-340. Medline 